\title{
Frequency anomaly in the Rashba-effect induced magnetization oscillations of a high-mobility two-dimensional electron system
}

\author{
B. Rupprecht, ${ }^{1}$ S. Heedt, ${ }^{2}$ H. Hardtdegen, ${ }^{2}$ Th. Schäpers, ${ }^{2}$ Ch. Heyn, ${ }^{3}$ M. A. Wilde,,${ }^{1, *}$ and D. Grundler ${ }^{1}$ \\ ${ }^{1}$ Lehrstuhl für Physik funktionaler Schichtsysteme, Technische Universität München, Physik Department, James-Franck-Strasse 1, \\ D-85747 Garching b. München, Germany \\ ${ }^{2}$ Peter Grünberg Institut (PGI-9) and JARA-FIT Jülich-Aachen Research Alliance, Forschungszentrum Jülich, D-52425 Jülich, Germany \\ ${ }^{3}$ Institut für Angewandte Physik und Zentrum für Mikrostrukturforschung, Universität Hamburg, D-20355 Hamburg, Germany
}

(Received 22 December 2010; revised manuscript received 29 November 2012; published 16 January 2013)

\begin{abstract}
With the direct measurement of the quantum oscillatory magnetization $M$ of a two-dimensional electron system (2DES) in an InGaAs/InP asymmetric quantum well we discover a frequency anomaly of the de Haas-van Alphen effect which is not consistent with existing theories on spin-orbit interaction (SOI). Strikingly, the oscillatory magnetoresistance of the same heterostructure, that is, the Shubnikov-de Haas effect conventionally used to explore SOI, does not show the frequency anomaly. This explains why our finding has not been reported for almost three decades. The understanding of the ground state energy of a 2DES is evidenced to be incomplete when SOI is present.
\end{abstract}

DOI: 10.1103/PhysRevB.87.035307

PACS number(s): 73.21.Fg, 75.70.Tj, 85.75.-d

\section{INTRODUCTION}

The spin-orbit interaction (SOI) in semiconductor heterostructures has received great interest due to the potential application in the field of spintronics on the one hand and the rich fundamental physics unveiled on the other hand. ${ }^{1}$ Electron momentum and spin couple due to an electric field provided by either an asymmetry of the confining potential or the inversion asymmetry of the host crystal. ${ }^{2}$ These phenomena are known as Rashba (R) and Dresselhaus (D) SOI, respectively. Beating patterns in the longitudinal magnetoresistivity $\rho_{x x}(B)$, that is, the Shubnikov-de Haas ( $\mathrm{SdH})$ effect, have often been evaluated to determine the strength of SOI in two-dimensional electron systems (2DESs). ${ }^{3,4}$ A transverse magnetic field $B$ hybridizes the SOI-induced zero-field splitting and the field dependent Zeeman splitting thus leading to two unequally spaced sets of Landau levels (LLs) that give rise to the beating patterns. Thermodynamic quantities like the magnetization $M$ are in particular powerful to study electronic states since they allow for an explicitly quantitative analysis and provide fundamental insight without further assumptions. ${ }^{5,6}$ Bychkov and Rashba have addressed $M$ of a 2DES with R-SOI in a pioneering theoretical work in $1984 .^{7}$ At zero temperature $T$ the oscillatory behavior of $M=-\partial U /\left.\partial B\right|_{n_{s}, T=0}$, that is, the de Haas-van Alphen (dHvA) effect, provides direct access to the evolution of the ground state energy $U$ with $B . n_{s}$ is the carrier density. Since then numerous theoretical papers have discussed the beating patterns in dHvA traces. ${ }^{8}$ Experimental data $M(B)$ in transverse magnetic fields to verify the theories have not yet been available due to the challenging magnetometry.,

In this paper we report on beating patterns in $M(B)$ measured at low temperature in nearly perpendicular and tilted magnetic fields on a 2DES in an asymmetric InGaAs/InP quantum well exhibiting SOI. In addition, we have taken data $\rho_{x x}$ vs $B$ on the same heterostructure under the same tilt angles. The experimental data evidence a clear anomaly in the frequency of the magnetization oscillations at nearly perpendicular $B$. The $\mathrm{SdH}$ oscillations do not exhibit the anomaly explaining why it has not been reported before. We show that this phenomenon is unexpected and in contrast to the current understanding. In particular we perform a fully quantum mechanical model calculation for $M(B)$ taking into account R-SOI, D-SOI, the Zeeman contribution, and tilted magnetic fields. The striking discrepancies between the magnetization data and both magnetotransport experiments and theoretical results reveal that the thermodynamic properties are far from being understood. We find that by tilting the field $B$ we are able to suppress the anomaly in $M(B)$.

\section{EXPERIMENT}

The measurements were performed on asymmetric $10 \mathrm{~nm}$ wide $\mathrm{Ga}_{0.23} \mathrm{In}_{0.77} \mathrm{As}$ quantum wells embedded between $150 \mathrm{~nm} \mathrm{Ga}{ }_{0.47} \mathrm{In}_{0.53}$ As and a $20 \mathrm{~nm} \mathrm{InP}$ spacer, followed by $10 \mathrm{~nm}$ InP:Si. The samples were grown by metal organic vapor phase epitaxy on $\operatorname{InP}(100)$. All measurements were taken after brief illumination with a blue light emitting diode. Electrons occupied only the lowest subband with a density $n_{s}=8.7 \times$ $10^{15} \mathrm{~m}^{-2}$ and mobility $\mu=37 \mathrm{~m}^{2} / \mathrm{V} \mathrm{s}$ at $T=300 \mathrm{mK}$. The effective Landé factor $g^{*}=-4.5$ and the effective mass $m^{*}=$ $0.037 m_{\mathrm{e}}$ were determined from tilted field measurements and the temperature dependence of quantum oscillations, ${ }^{11}$ respectively ( $m_{\mathrm{e}}$ is the free-electron mass). We have employed a micromechanical cantilever magnetometer ${ }^{12}$ to measure the anisotropic magnetization $\mathbf{M}$ of the 2DES. The experimental geometry is sketched in the inset of Fig. 1. $M$ was determined by measuring the torque $\boldsymbol{\tau}=\mathbf{M} \times \mathbf{B}$ using a capacitive readout scheme. The oscillatory part of $M$ was extracted from the raw data by fitting and subtracting a low-order polynomial in $1 / B .^{12,13}$ The angle $\theta$ was varied in situ. Magnetization data were recorded at $T=30$ and $300 \mathrm{mK}$. We found the data to be consistent. $\rho_{x x}$ was measured on a Hall bar from the same heterostructure at $T=300 \mathrm{mK}$ using current modulation and phase-sensitive detection with a lock-in amplifier. By this we revisited the magnetotransport experiments used earlier to investigate SOI in high-mobility 2DESs. ${ }^{3,4}$

\section{RESULTS AND DISCUSSION}

We present experimental magnetization data for different $\theta$ in Fig. 1(a). Data are displayed vs $1 / B_{\perp}$ for clarity. Here $B_{\perp}$ is the magnetic field component perpendicular to the 2DES. Due 

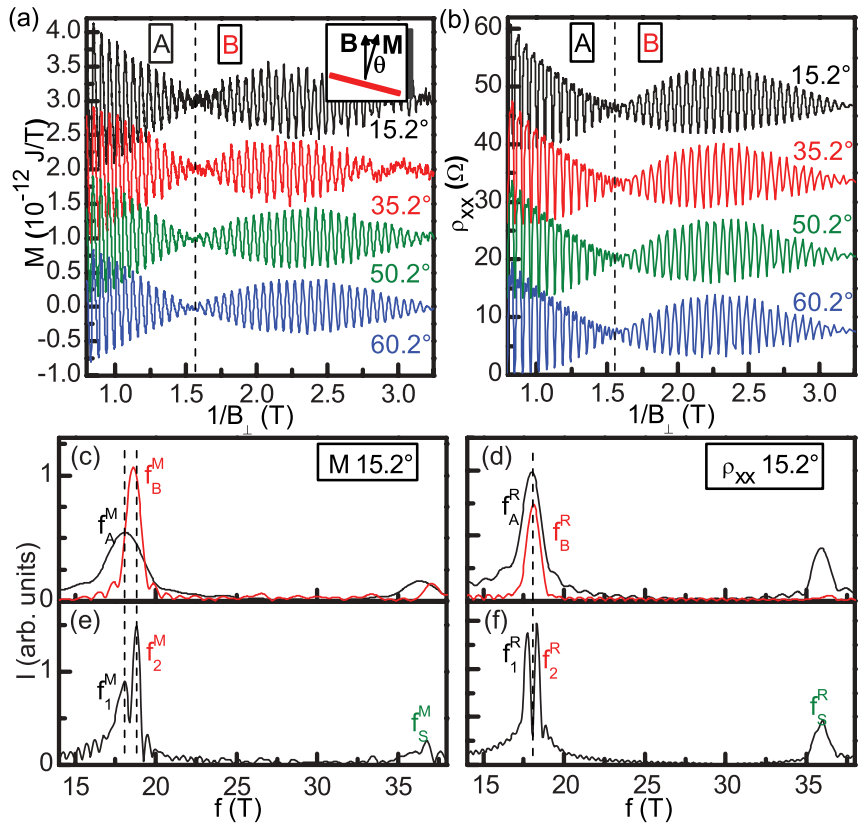

FIG. 1. (Color online) (a) Magnetization $M\left(1 / B_{\perp}\right)$ measured for different tilt angles $\theta$. Data are taken at $30 \mathrm{mK}$ and are offset for clarity. Inset: Schematic side view of the heterostructure [light (red)] in the external magnetic field B. (b) $\rho_{x x}\left(1 / B_{\perp}\right)$ measured under corresponding tilt angles. Curves are taken at $300 \mathrm{mK}$ and are offset for clarity. Vertical lines in (a) and (b) indicate the last beat node and separate the field regimes A and B used in the data analysis. (c) FFT spectra of $M$ taken separately on the field regimes $\mathrm{A}$ and $\mathrm{B}$ provide two distinctly different oscillation frequencies $f_{\mathrm{A}}^{M}$ and $f_{\mathrm{B}}^{M}$, respectively. In contrast, (d) FFT spectra taken separately on the field regimes A and $\mathrm{B}$ in $\rho_{x x}$ yield peaks with $f_{\mathrm{A}}^{\mathrm{R}}$ and $f_{\mathrm{B}}^{\mathrm{R}}$ being almost exactly the same. (e) Applying an FFT on $M$ data on the full field regime A + B provides two different frequencies $f_{1}^{M}$ and $f_{2}^{M}$ as well as the sum frequency $f_{\mathrm{S}}^{M}=f_{1}^{M}+f_{2}^{M}$. (f) Full range FFT of the $\rho_{x x}$ data provides also two different frequencies $f_{1}^{\mathrm{R}}$ and $f_{2}^{\mathrm{R}}$ as well as the sum frequency $f_{\mathrm{S}}^{\mathrm{R}}=f_{1}^{\mathrm{R}}+f_{2}^{\mathrm{R}}$.

to SOI, the oscillations exhibit a characteristic beating pattern first predicted for $M\left(1 / B_{\perp}\right)$ by Bychkov and Rashba. ${ }^{7}$ At $\theta=$ $15.2^{\circ}$ the last beat node is found at $1 / B_{\perp} \simeq 1.6 / \mathrm{T}$. For large $\theta$, the second beat node at $1 / B_{\perp} \simeq 3.25 / \mathrm{T}$ is resolved due to the higher experimental resolution caused by the increased torque $\tau=M B \sin \theta$. It is well established that the sharply dropping slopes of the sawtoothlike dHvA effect occur at integer values of the filling factor $v=n_{s} /\left(e B_{\perp} / h\right)$, where the Fermi energy resides between the LLs. ${ }^{13,14}$ The jumps in $M$ have a peakto-peak amplitude $\Delta M$ and measure the energy gap between adjacent levels according to $\Delta E=\Delta M B / N$, where $N$ is the number of charge carriers. ${ }^{13,15}$

Before we analyze in detail the magnetization data, it is instructive to consider the $\mathrm{SdH}$ oscillations in $\rho_{x x}\left(1 / B_{\perp}\right)$ [Fig. 1(b)] measured on the same heterostructure. They exhibit the well-known SOI-induced beating pattern as well. Here the minima in $\rho_{x x}$ are known to reflect integer values of $v$. From the comparison between Figs. 1(a) and 1(b) we find that the beat node positions in $\rho_{x x}$ and $M$ are at corresponding field values (taking into account a small carrier density difference of $2 \%$ ). At first sight, the overall behaviors of $M$ and $\rho_{x x}$ seem to be consistent. This is, however, not the case when considering the frequency of the oscillations as a function of $1 / B_{\perp}$.

To show this, we first focus on the well-established evaluation based on fast Fourier transformation $(\mathrm{FFT})^{3,4,16}$ of $M$ and $\rho_{x x}$ data. We consider $M$ and $\rho_{x x}$ at $\theta=15.2^{\circ}$ in Figs. 1(a) and 1(b), respectively, and subdivide the data sets into regimes $\mathrm{A}$ and $\mathrm{B}$ as indicated. Performing FFTs in the two regimes separately, we obtain two peaks $\mathrm{A}$ at $f_{\mathrm{A}}^{M}=18.1 \mathrm{~T}$ and $\mathrm{B}$ at $f_{\mathrm{B}}^{M}=18.6 \mathrm{~T}$ from the magnetization data [Fig. 1(c)]. The occurrence of two separate frequency peaks is unexpected and in contrast to the FFTs performed on the magnetoresistance $(\mathrm{R})$ data. In Fig. 1(d) we find the same frequency for regimes $\mathrm{A}$ and $\mathrm{B}$, that is, $f_{\mathrm{A}}^{\mathrm{R}} \approx f_{\mathrm{B}}^{\mathrm{R}} \approx 18 \mathrm{~T}$. The degeneracy of these frequencies is expected for a regular beating pattern and has been reported consistently for $\rho_{x x}$ for decades, where oscillations between two beat nodes exhibit only one single periodicity. The important observation of Figs. 1(c) and 1(d) is that we obtain a clear frequency anomaly for the magnetization data that are taken on the same heterostructure. Applying FFTs over the full magnetic field range $\mathrm{A}+\mathrm{B}$ [Figs. 1(e) and 1(f)], we obtain peaks at separate frequencies for both experiments. We find $f_{1}^{M}=18.1 \mathrm{~T}$ and $f_{2}^{M}=18.8 \mathrm{~T}$ for $M$, and $f_{1}^{\mathrm{R}}=17.7 \mathrm{~T}$ and $f_{2}^{\mathrm{R}}=18.3 \mathrm{~T}$ for $\rho_{x x}$. Following the vertical dashed lines in Figs. 1(c)-1(f) it is easy to see that in the magnetization data $f_{\mathrm{A}}^{M}=f_{1}^{M}$ and $f_{\mathrm{B}}^{M}=f_{2}^{M}$, whereas in the transport data, $f_{\mathrm{A}}^{\mathrm{R}}=f_{\mathrm{B}}^{\mathrm{R}}=$ $\left(f_{1}^{\mathrm{R}}+f_{2}^{\mathrm{R}}\right) / 2$. This discrepancy further substantiates that the oscillations in $M$ exhibit an anomaly compared to $\rho_{x x}$. Using $\alpha=\left(\Delta n \hbar^{2} / m^{*}\right)\left[\pi / 2\left(n_{s}-\Delta n\right)\right]^{1 / 2}$ from Ref. 4, both FFTs over the full range yield very similar values for the Rashba constant $\alpha_{R}$ of $4.6 \times 10^{-12} \mathrm{eV}$ m for $M$ and $4.1 \times 10^{-12} \mathrm{eV} \mathrm{m}$ for $\rho_{x x} \cdot{ }^{17}$

To gain further insight into the frequency anomaly in $M$, we plot integer $v$ versus $1 / B_{\perp}$ for each oscillation as open symbols in Fig. 2(a). Analyzing these positions for $M$, we find two different slopes for the data after and before the last beat node. The slopes correspond to the frequencies $2 \cdot f_{\mathrm{A}}^{M}$ (dashed line) and $2 \cdot f_{\mathrm{B}}^{M}$ (dash-dotted line), respectively. The sum frequency $\left(f_{\mathrm{A}}^{M}+f_{\mathrm{B}}^{M}\right)$ is not relevant to describe the experimental data $M(B)$. This finding is in contrast to the transport data. The oscillations in $\rho_{x x}$ [symbols in Fig. 2(b)] strictly follow the sum frequency $\left(f_{\mathrm{A}}^{\mathrm{R}}+f_{\mathrm{B}}^{\mathrm{R}}\right)=f_{\mathrm{S}}^{\mathrm{R}}$ (solid line). Here no change in slope is found around the last beat node. For decades, the slope of $v$ vs $1 / B_{\perp}$ has been interpreted in terms of $(h / e) n_{s}$, thereby providing the carrier density $n_{s}$ of the $2 \mathrm{DES}$. Following this, we now define the parameter $n_{s}^{*}=\left(e B_{\perp} / h\right) v$ and evaluate this parameter in Fig. 2(c) for both the magnetization and transport data of Figs. 1(a) and 1(b), respectively. For $\rho_{x x}$, the parameter $n_{s}^{*}$ is found to be constant within the error bar detailed below. By interpreting $M$ of Fig. 1(a) in the same way, the parameter $n_{s}^{*}$ is found to jump near the last beat node at $\theta=15.2^{\circ}$ in Fig. 2(c) (uppermost graph). ${ }^{18}$ The error bar of $n_{s}^{*}$ is derived as follows: For $\rho_{x x}$, it is well known that $n_{s}^{*}=n_{s}=$ const. for all fields and angles $\theta$. The variation in $\rho_{x x}$ data in Fig. 2(c) thus reflects the error for $n_{s}^{*} .{ }^{19}$ The maximum peakto-peak error amounts to $\delta n_{s}^{*}=(8.68-8.52) \times 10^{15} / \mathrm{m}^{2}=$ $0.18 \times 10^{15} / \mathrm{m}^{2}$, that is, $2.1 \%$. At $\theta=15.2^{\circ}$, the jump in $n_{s}^{*}$ extracted from $M(B)$ amounts to $5.2 \%$, being far larger than the maximum error in $n_{s}^{*}$. We have studied the striking discrepancy between the transport and magnetization data 

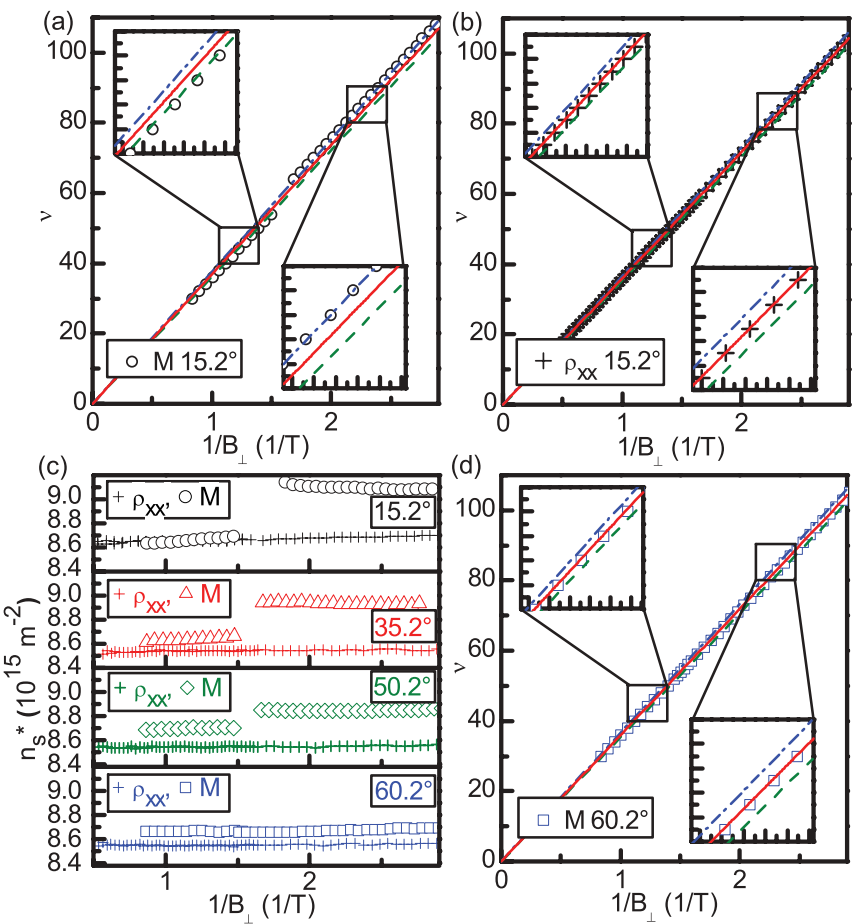

FIG. 2. (Color online) (a) Integer $v$ vs $1 / B_{\perp}$ for $M$ taken at $\theta=15.2^{\circ}$. Insets highlight that oscillations before and after the last beat node follow two different slopes. Note that the fitted linear slopes directly correspond to the frequency peaks $2 \cdot f_{\mathrm{A}}^{M}$ (dashed line) and $2 \cdot f_{\mathrm{B}}^{M}$ (dash-dotted line) extracted from Fig. 1(c). Strikingly, they do not follow the sum frequency (solid line) in contrast to the orthodox understanding. (b) For $\rho_{x x}$ and $\theta=15.2^{\circ}$, integer $v$ vs $1 / B_{\perp}$ strictly follow the slope given by the sum frequency $f_{\mathrm{S}}^{\mathrm{R}}$ (solid line). (c) Parameter $n_{s}^{*}=\left(e B_{\perp} / h\right) v$ evaluated from the period of adjacent oscillations observed in $\rho_{x x}$ (crosses) and $M$ (open symbols). In $\rho_{x x}$ we get a constant behavior at each angle, consistent with the orthodox theory. At a fixed angle, the remaining variation in $n_{s}^{*}$ indicates the error bar of the evaluation method. When we evaluate $n_{s}^{*}$ for $M$ vs $B_{\perp}$, we find an unexpected jump in $n_{s}$ for $\theta<60^{\circ}$ which depends on $\theta$. This jump highlights the frequency anomaly discovered for $M(B)$. Data points are missing at the node positions because oscillations in $M(B)$ were vanishingly small and $v$ vs $1 / B_{\perp}$ was not evaluated there. At $\theta=60.2^{\circ}, n_{s}^{*}$ is constant for $M$, and (d) the slope in $v$ corresponds to the sum frequency.

towards higher tilt angles. For $\rho_{x x}, n_{s}^{*}$ is constant within the error bar at all tilt angles. For $M$, we find a constant $n_{s}^{*}$ only at large $\theta \geqslant 60.2^{\circ}$. The jump is found to vanish at $\theta=60.2^{\circ}$ [bottom-most graph in Fig. 2(c)]. Correspondingly, $v$ follows strictly the sum frequency in Fig. 2(d). Here $n_{s}^{*}$ is consistent with a constant (field-independent) $n_{s}$. The ground-state property $M(B)$ thus behaves strikingly different compared to $\rho_{x x}$. We have ruled out experimental artifacts by repeated measurements on different samples in two setups and in several cooling cycles. All data sets are consistent, suggesting that there is a fundamental difference in the physics underlying the beatings in $\rho_{x x}$ and $M$. We now show that the anomaly in the frequency of $M$ vs $1 / B_{\perp}$ is completely unexpected also from the theoretical side and discuss possible origins.

To this end we develop a state-of-the-art numerical model for $M$ based on the one-electron Hamiltonian $H=$
$H_{0}+H_{R}+H_{D}$ taking into account the R-SOI term $H_{R}=$ $\frac{\alpha_{R}}{\hbar}\left(\sigma_{x} \pi_{y}-\sigma_{y} \pi_{x}\right)$, the $k$-linear D-SOI term $H_{D}=\frac{\beta_{D}}{\hbar}\left(\sigma_{x} \pi_{x}-\right.$ $\left.\sigma_{y} \pi_{y}\right)$, and the Zeeman term in tilted magnetic fields. Here $H_{0}=\frac{\pi^{2}}{2 m^{*}}+\frac{1}{2} g^{*} \mu_{\mathrm{B}} \mathbf{B} \boldsymbol{\sigma}\left(\pi_{x, y}\right.$ are the components of the kinetic momentum operator, $\boldsymbol{\sigma}$ is the vector of the Pauli matrices, and $\mu_{\mathrm{B}}$ is the Bohr magneton). Following Ref. 20 the eigenvalues are determined by evaluating the matrix elements of $H$ between the eigenstates of $H_{0}$ in perpendicular magnetic fields. The resulting matrix is diagonalized numerically by truncating the matrix dimensions while including a sufficient number of LLs. Calculations use the values for $g^{*}$ and $m^{*}$ determined from the experiment. $\alpha_{R}$ and $\beta_{D}$ are used as parameters to fit the experiment. Using the energy eigenvalues $\epsilon_{i}$ the position of the Fermi energy $E_{F}$ is determined from $n_{s}=\int_{-\infty}^{E_{F}} D(E) d E$, with the density of states (DOS) $D(E)=\sum_{i} 1 /(\sqrt{2 \pi} \Gamma) \exp \left[-\frac{\left(E-\epsilon_{i}\right)^{2}}{2 \Gamma^{2}}\right]$. Here the level broadening $\Gamma \propto \sqrt{B}$ was chosen to match the experimental oscillation amplitudes. The ground state energy $U$ is then calculated from $D(E)$, and the magnetization is obtained through $M=-\partial U /\left.\partial B\right|_{n_{s}, T=0} .^{21}$

We now show in Figs. 3(a) and 3(b) that the experimental data (black) at $\theta=60.2^{\circ}$ can be modeled consistently assuming a dominant R-SOI contribution. To illustrate this, a calculation assuming $H=H_{0}+H_{D}$ [light (blue) line] is shown in Fig. 3(a). $\Delta_{D}=2.4 \mathrm{meV}$ was chosen to match the position of the last beat node. We find that the amplitude evolution as well as the position of the second last beat node cannot be modeled assuming $H=H_{0}+H_{D}$. In Fig. 3(b) we consider $H=H_{0}+H_{R}$ [light (red) line]. $\alpha_{R}$ was adjusted to match the last node position. The amplitudes of the magnetization oscillations as well as the position of the second last beat node are well modeled for the full field regime taking
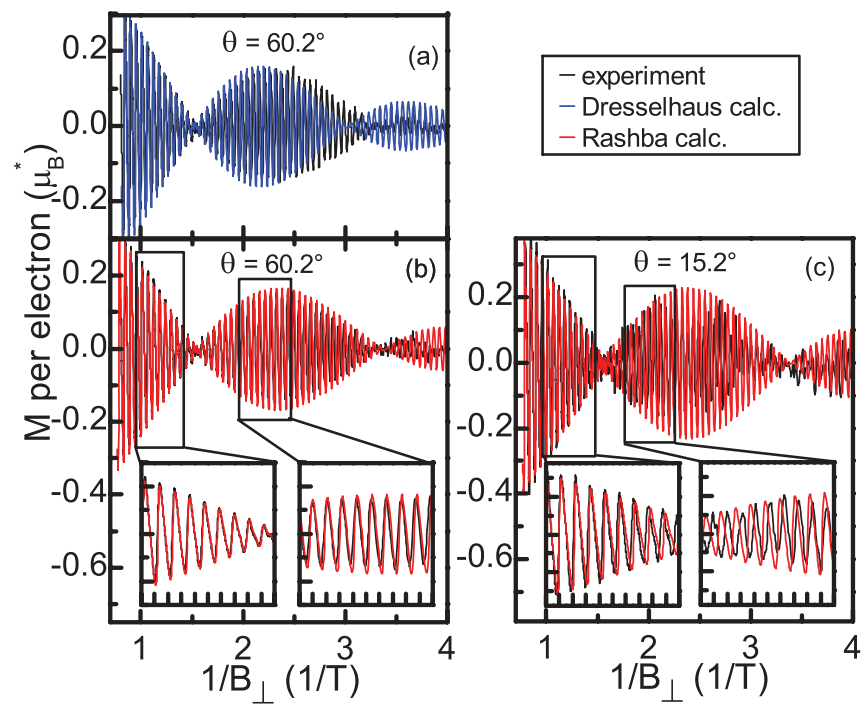

FIG. 3. (Color online) (a) Experimental data for $\theta=60.2^{\circ}$ (black) and D-SOI calculation [light (blue)]. (b) Data as in (a), but with R-SOI calculation [light (red)]. The insets highlight the excellent reproduction of oscillation amplitude and frequency by the R-SOI theory. (c) Experimental data for $\theta=15.2^{\circ}$ (black) with R-SOI calculation [light (red)]. The bottom-right inset shows the discrepancy between the constant frequency in the calculation and the frequency anomaly in the experimental data. 
$\alpha_{R}=4.5 \times 10^{-12} \mathrm{eV} \mathrm{m}$. The oscillation frequency is well reproduced as demonstrated by the insets for the regimes after and before the last beat node for $\theta=60.2^{\circ}$. In the calculation, oscillations occur always at integer $v$ since only the total density $n_{s}$ and the level degeneracy $e B_{\perp} / h$ enter in the position of integer $v{ }^{22}$ For $\theta=15.2^{\circ}$ there is however a discrepancy between the experimental data and the calculation also for $H=H_{0}+H_{R}$. In Fig. 3(c) only the position of the last beat node and the oscillation amplitudes are well reproduced for $\alpha_{R}=4.5 \times 10^{-12} \mathrm{eV} \mathrm{m}$ but not the frequency. This can be seen from the insets in Fig. 3(c). In the bottom-left inset, the experimental oscillation still follows closely the calculated trace. In the bottom-right inset, however, the experimental data are found to oscillate at a higher frequency.

Using the state-of-the-art theoretical approach based on the current understanding of SOI, we are able to reproduce fine details in the beating patterns of $M(B)$. The only experimental observation that is not captured by the model is the frequency anomaly occurring at tilt angles $\theta<60^{\circ}$. Since $M$ is linked to the model Hamiltonian in a straightforward way, our finding of the anomaly raises the fundamental question whether the current picture of SOI-induced beating patterns in magneticfield dependent quantum oscillations is comprehensive. The origin of both the frequency anomaly and its evolution with tilt angle $\theta$ is unknown at present. In the following we speculate about possible origins.

The data seem to suggest that magnetic quantum oscillations of one carrier subset are seen in the low field regime, whereas in the high field regime oscillations of a subset with a different density are seen. However, a straightforward association of the frequencies $f_{\mathrm{A}}^{M}$ and $f_{\mathrm{B}}^{M}$ with the two spin subsets present in the 2DES is not reasonable since the frequency difference between $f_{\mathrm{A}}^{M}$ and $f_{\mathrm{B}}^{M}$ vanishes with increasing tilt angle [Fig. 2(c)], while, strikingly, the beating pattern itself remains unaffected. How can two different frequencies (densities) appear in the two regimes? It has been put forward that the quantum relaxation times might differ between the two unequally populated spin subsets, ${ }^{3,23,24}$ thus potentially leading to preferential observation of only one subset in a given field regime. We have modeled $M$ including a subset-dependent relaxation time and find that this cannot account for the experimental observations.
In a recent approach based on the Gutzwiller trace formula, ${ }^{2,25}$ anomalous magneto-oscillations reflect the nonadiabatic spin precession along the cyclotron orbits. However, the work focused on the analysis of Fourier transforms of $\rho_{x x}$. Predictions for the periodicity of oscillations in the thermodynamic quantity $M$ were not made.

A fundamental difference between $M$ and $\rho_{x x}$ is that $M$ is determined by the complete DOS of the electron system, including the LLs below $E_{F}$, while the resistance depends on microscopic details of the scattering ${ }^{26}$ and probes $D\left(E_{F}\right)$, that is, it does not monitor the lower-lying energy levels. Thus, an interaction-induced renormalization of energy levels ${ }^{27}$ lying below $E_{F}$ could lead to differences in $M(B)$ and $\rho_{x x}(B)$. In combination with R-SOI-induced avoided crossings of low-lying LLs in tilted magnetic fields, ${ }^{28}$ interaction effects might also contribute to an angular dependence of the anomalous behavior of $M$. A theoretical treatment considering such many-body aspects is beyond the scope of the present paper.

\section{CONCLUSION}

In conclusion, we have reported a frequency anomaly in the SOI-modified magneto-oscillations of $M$ that is absent in $\rho_{x x}$ and that is not captured by the well accepted stateof-the-art quantum mechanical description. This is especially intriguing since $M$ is a thermodynamic quantity that canunlike $\rho_{x x}$ - be calculated in a straightforward way from the energy levels and the ground state energy $U$. The evaluation of quantum oscillations is a widespread method to explore SOI-induced properties of 2DESs. We expect our results to stimulate further theoretical and experimental investigations into this fundamental subject for spintronics.

\section{ACKNOWLEDGMENTS}

We thank E. I. Rashba and R. Winkler for valuable discussions and D. Heitmann and W. Hansen for experimental help. We gratefully acknowledge financial support by the DFG via SPP1285 "Halbleiter-Spintronik," Grant No. GR1640/3, and the German Excellence Initiative via Nanosystems Initiative Munich (NIM). *mwilde@ph.tum.de

${ }^{1}$ D. D. Awschalom, D. Loss, and N. Samarth, Eds., Semiconductor Spintronics and Quantum Computation (Springer Nanoscience and Technology, Berlin, 2002); D. Grundler, Phys. World 15, 39 (2002).

${ }^{2} \mathrm{R}$. Winkler, Spin-Orbit Coupling Effects in Two-Dimensional Electron and Hole Systems (Springer, Berlin, 2003).

${ }^{3}$ B. Das, D. C. Miller, S. Datta, R. Reifenberger, W. P. Hong, P. K. Bhattacharya, J. Singh, and M. Jaffe, Phys. Rev. B 39, R1411 (1989); J. Luo, H. Munekata, F. F. Fang, and P. J. Stiles, ibid. 41, 7685 (1990); J. Nitta, T. Akazaki, H. Takayanagi, and T. Enoki, Phys. Rev. Lett. 78, 1335 (1997); D. Grundler, ibid. 84, 6074 (2000).

${ }^{4}$ G. Engels, J. Lange, T. Schäpers, and H. Lüth, Phys. Rev. B 55, R1958 (1997).
${ }^{5}$ H. Weiss, M. V. Kartsovnik, W. Biberacher, E. Balthes, A. G. M. Jansen, and N. D. Kushch, Phys. Rev. B 60, R16259 (1999).

${ }^{6}$ N. Ruhe, J. I. Springborn, C. Heyn, M. A. Wilde, and D. Grundler, Phys. Rev. B 74, 235326 (2006).

${ }^{7}$ Y. A. Bychkov and E. I. Rashba, J. Phys. C 17, 6039 (1984).

${ }^{8}$ E. A. de Andrada e Silva, G. C. La Rocca, and F. Bassani, Phys. Rev. B 50, 8523 (1994); W. Zawadzki and P. Pfeffer, Physica E 13, 533 (2002); Z. Wang, W. Zhang, and P. Zhang, Phys. Rev. B 79, 235327 (2009).

${ }^{9}$ A. Usher and M. Elliott, J. Phys.: Condens. Matter 21, 103202 (2009).

${ }^{10} \mathrm{M}$. A. Wilde, D. Heitmann, and D. Grundler, Magnetization of Interacting Electrons in Low-Dimensional Systems (Springer Nanoscience and Technology, Berlin, 2010), Chap. 10, p. 245. 
${ }^{11}$ T. Schäpers, G. Engels, J. Lange, T. Klocke, M. Hollfelder, and H. Lüth, J. Appl. Phys. 83, 4324 (1998).

${ }^{12}$ M. A. Wilde, J. I. Springborn, O. Roesler, N. Ruhe, M. P. Schwarz, D. Heitmann, and D. Grundler, Phys. Status Solidi B 245, 344 (2008).

${ }^{13}$ J. P. Eisenstein, H. L. Stormer, V. Narayanamurti, A. Y. Cho, A. C. Gossard, and C. W. Tu, Phys. Rev. Lett. 55, 875 (1985); S. A. J. Wiegers, M. Specht, L. P. Lévy, M. Y. Simmons, D. A. Ritchie, A. Cavanna, B. Etienne, G. Martinez, and P. Wyder, ibid. 79, 3238 (1997); M. A. Wilde, D. Reuter, C. Heyn, A. D. Wieck, and D. Grundler, Phys. Rev. B 79, 125330 (2009).

${ }^{14}$ M. A. Wilde, M. P. Schwarz, C. Heyn, D. Heitmann, D. Grundler, D. Reuter, and A. D. Wieck, Phys. Rev. B 73, 125325 (2006).

${ }^{15}$ A. H. MacDonald, H. C. A. Oji, and K. L. Liu, Phys. Rev. B 34, 2681 (1986); I. Meinel, D. Grundler, D. Heitmann, A. Manolescu, V. Gudmundsson, W. Wegscheider, and M. Bichler, ibid. 64, 121306 (2001).

${ }^{16}$ R. A. Shepherd, M. Elliott, W. G. Herrenden-Harker, M. Zervos, P. R. Morris, M. Beck, and M. Ilegems, Phys. Rev. B 60, R11277 (1999).

${ }^{17} n_{s}$ and $\Delta n$ are determined from the FFT frequencies via $n_{s}=$ $\left(f_{S}^{Y} \cdot e\right) / h, \Delta n=\left[\left(f_{2}^{Y}-f_{1}^{Y}\right) \cdot e\right] / h$ with $Y=M, R$.
${ }^{18}$ The jump is found to be present in $M$ of the illuminated and unilluminated (not shown) 2DESs with SOI.

${ }^{19}$ The angular variation of $n_{s}^{*}$ is attributed to the small uncertainty remaining in $\theta$. The transport data have been obtained on a separate 2DES from the same heterostructure in a separate cool-down and illumination step. This accounts for the small difference in $n_{s}^{*}$ for $\rho_{x x}$ and $M$ at $\theta=60.2^{\circ}$.

${ }^{20}$ B. Das, S. Datta, and R. Reifenberger, Phys. Rev. B 41, 8278 (1990).

${ }^{21} \mathrm{We}$ checked the influence of finite temperature and found it to be negligible in the range considered here.

${ }^{22}$ S. G. Novokshonov and A. G. Groshev, Phys. Rev. B 74, 245333 (2006).

${ }^{23}$ W. Xu, Phys. Rev. B 71, 245304 (2005).

${ }^{24}$ A. M. Gilbertson, W. R. Branford, M. Fearn, L. Buckle, P. D. Buckle, T. Ashley, and L. F. Cohen, Phys. Rev. B 79, 235333 (2009).

${ }^{25}$ S. Keppeler and R. Winkler, Phys. Rev. Lett. 88, 046401 (2002).

${ }^{26}$ M. Langenbuch, M. Suhrke, and U. Rössler, Phys. Rev. B 69, 125303 (2004).

${ }^{27}$ C. H. Yang and W. Xu, J. Appl. Phys. 103, 013707 (2008).

${ }^{28}$ Yu. A. Bychkov, V. I. Mel'nikov, and E. I. Rashba, Sov. Phys. JETP 71(2), 401 (1990). 